\title{
Secretome-microRNA and anti-proliferative APRO family proteins as cancer prevention and stem cell research strategies
}

\author{
Yuka IKEDA; Nozomi NAGASE; Ai TSUJI; Kurumi TANIGUCHI; Yasuko KITAGISHI; Satoru MATSUDA* \\ Department of Food Science and Nutrition, Nara Women's University, Nara, 630-8506, Japan
}

Key words: Cancer, Stem cell, Secretome, Proliferation, APRO family, microRNA

\begin{abstract}
Stemness of cancer cells contains limitless self-renewal proliferation. For the purpose of proliferation, secretome might exert its effects via the paracrine signaling. Specific microRNAs enclosed in the secretome of cancer stem cells could regulate the expression of anti-proliferative APRO family proteins. The biological functions of APRO family proteins seems to be quite intricate, however, which might be a key modulator of microRNAs, then could regulate the proliferation of cancer cells. In addition to affecting proliferation/differentiation during cellular development, APRO family proteins might also play an imperious role on keeping homeostasis in healthy stem cells under a physiological condition. Therefore, relationship between the microRNAs and the APRO family proteins has attracted much attention in the field of cancer research and regenerative medicine. Here, we have described the molecular mechanism behind this interplay that have a potential role in the future promising tools with targeting APRO family proteins for the medical applications.
\end{abstract}

$\begin{array}{ll}\text { Abbreviations } \\ \text { APRO: } & \text { antiproliferative protein } \\ \text { BTG: } & \text { B cell translocation gene } \\ \text { HSCs: } & \text { hematopoietic stem cells } \\ \text { miRNAs: } & \text { microRNAs } \\ \text { mRNA: } & \text { messenger RNA } \\ \text { MSCs: } & \text { mesenchymal stem/stromal cells } \\ \text { ROS: } & \text { reactive oxygen species } \\ \text { TOB: } & \text { transducer of ErbB2 }\end{array}$

\section{Introduction}

Stem cells including mesenchymal stem/stromal cells (MSCs) and hematopoietic stem cells (HSCs) are promising tools for regenerative medicine applications (Priester et al., 2020). Stemness properties of the stem cells include limitless selfrenewal proliferation and the ability to differentiate into all cell-types in a body (Barati et al., 2021). Similarly, cancer stem cells also exhibit limitless self-renewal proliferation, which is eventually responsible for resistance to cancertherapy and/or for cancer-relapse (Lang et al., 2021, Treisman, Li and Zhu, 2021). Obtaining the ability of

\footnotetext{
*Address correspondence to: Satoru Matsuda, smatsuda@cc.nara-wu.ac.jp Received: 05 July 2021; Accepted: 22 September 2021
}

infinite proliferation, cancer cells may become immortalized. In the meaning of self-renewal avoiding exhaustion, stem cells have to proliferate by themselves at least to keep their population with functioning stem cells (Fig. 1). For the selfrenewal proliferation, quiescent initial stem cells should be stimulated by the proliferation-signaling from either outside or inside of the stem cells. Reactive oxygen species (ROS) may have physiological roles as signaling molecules that regulate stem cells function (Matsuda et al., 2018). In particular, redox-mediated regulation has significant implications for stem cell biology particularly in the hypoxic situation of repair-injuries (Paul et al., 2014). In general, the stem cells in a hypoxic microenvironment exhibit low levels of ROS that may allow to proliferate and/or to protect stem cells, whereas intermediate levels of ROS may promote the differentiation of stem cells (Pasha et al., 2021). High levels of ROS may cause the senescence of stem cells or apoptosis/ cell-death. However, regulator mechanisms of the dynamism in different stem cells as well as downstream mechanisms about the homeostasis of stem cells are practically unclear. MSCs may secrete various cytokines and growth factors as well as extracellular vesicles termed secretome which contain microRNAs (miRNAs), mRNAs, and the cytokine/ growth-factor proteins (Basalova et al., 2020). Secretome of stem cells may exert its effects generally via the paracrine signaling (Lanfredi et al., 2021). Consistently, recent reports also suggest that the activity of stem cells is predominantly 


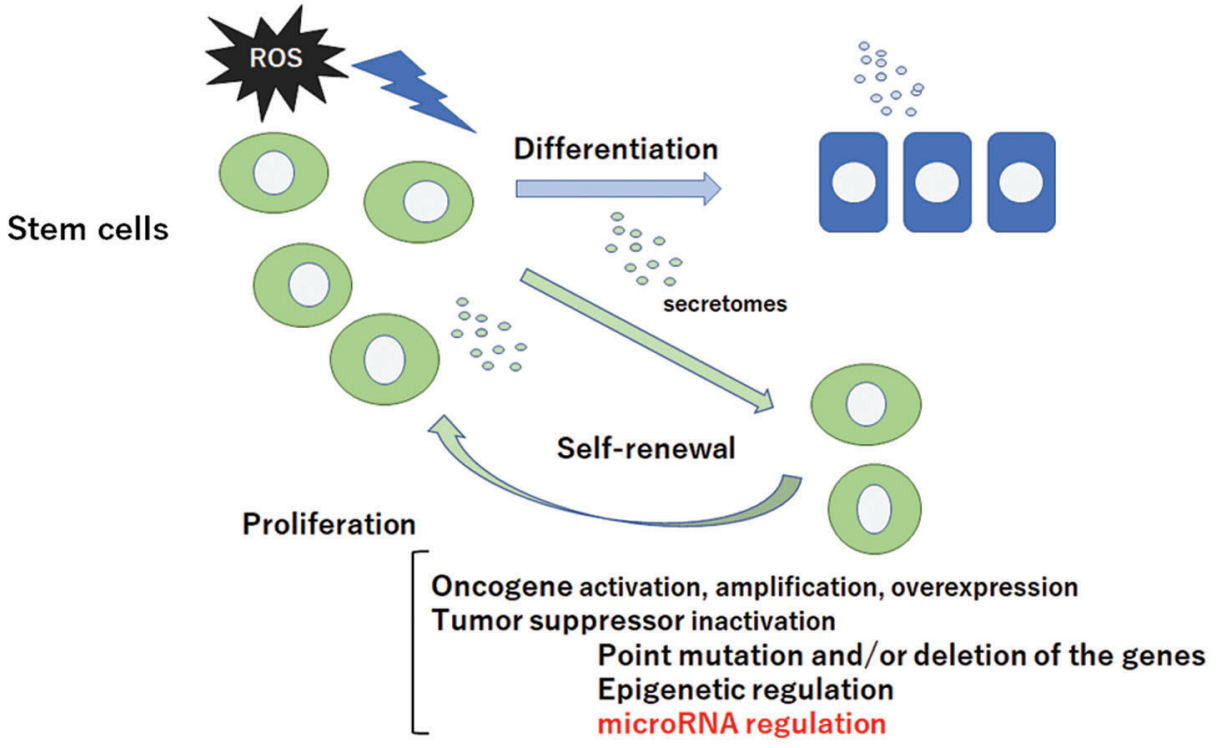

\begin{abstract}
FIGURE 1. Scheme of stem cells selfrenewal, proliferation, and differentiation. Stem cells are capable of maintaining the stem cells population through appropriate self-renewal and proliferation. Quiescent and/or self-renewing stem cells display low reactive oxygen species (ROS) levels probably due to their robust antioxidant ability. Intermediate levels of ROS may prime stem cells for differentiation. Tumor suppressor inactivation by microRNAregulation could lead to the proliferation of stem cells.
\end{abstract}

mediated by the paracrine effect of secretomes (Jiang et al., 2021). In fact, manipulation of stem cells-derived secretome has emerged as a novel alternative therapeutic option for several diseases and/or in regenerative medicine (Rajan et al., 2016). The miRNAs frequently being enclosed in secretome are endogenous single-strand non-encoding RNAs with the length of about 20 nucleotides, which are generally found in eukaryotic organisms (Bartel, 2009). The miRNAs can affect the stability and/or translational efficiency of their target mRNAs resulting in downregulated gene expression and reduced protein output (Mohr and Mott, 2015). More than 1,000 miRNAs have been revealed to regulate a series of biological processes including proliferation, differentiation, and apoptosis (Dexheimer and Cochella, 2020). By utilizing this miRNAs in secretome, stem cells might provide proliferative signaling each other for the maintenance of stemness in a stem cell pool. In fact, miR21 in a stem cell exosome could enhance the proliferation of cells (Yang et al., 2020; Li et al., 2018). In addition, miR590 found in exosome has been established to be a promoter of cell proliferation, migration and invasion in numerous cancer cells, as well as in human umbilical cord MSCs (Liu et al., 2017). Interestingly, miR21 regulates the expression of BTG2, a member of APRO family, suggesting that miR21 and the expression of BTG2 are negatively correlated during carcinogenesis (Mao et al., 2015a). Besides, miR590 exerts their effects through targeting TOB1, also a member of APRO family. Furthermore, miR511 in exosome promotes the proliferation of human hepatoma cells by targeting the 3'UTR mRNA of BTG1, also a member of APRO family (Zhang et al., 2017). In addition, miR32-5p was significantly upregulated in colorectal cancer tissues compared with adjacent normal tissues, which might regulate proliferation/ invasion of cancer stem cells through inhibiting and/or decreasing the expression of TOB1 in APRO family (Liang et al., 2019). Exosome containing miR486-5p has facilitated osteoblast differentiation by repressing TOB1 expression (Chen et al., 2020). In this way, the relationship between miRNAs and APRO family proteins has attracted much attention for the regulation of stem cell proliferation.
The APRO family anti-proliferative genes are characterized in immediate early growth responsive genes (Matsuda et al., 2001). The gene products include PC3/TIS21/BTG2, BTG1, TOB1, TOB2, ANA/BTG3, PC3B and others (Matsuda et al., 2001). These APRO family proteins have been described to participate in diverse human diseases such as cancers, which have also been implicated in a variety of cellular processes including celldivision, DNA repair, and mRNA stability (Yuniati et al., 2019). Accordingly, APRO family members consequently may be involved in physiological and/or pathological processes including cell proliferation, cell differentiation, apoptosis/programmed cell death, and acting as tumor suppressors (Yuniati et al., 2019). In fact, APRO protein expressions are frequently downregulated in many human cancers and in stem cells (Micheli et al., 2015). In addition, significant prognostic effects of APRO family members have been shown in defined cancer types (Yang, Shen \& Sun, 2018). As for stem cells, knockdown of TOB1 has increased proliferative activity of rat MSCs in vitro (Gao et al., 2016). The expression of TOB1 in MSCs may also be regulated by miR218 (Gao et al., 2016). In addition, TOB1 and TOB2 could inhibit proliferation of mouse embryonic stem cells, which does not compromise the basic properties of stem cells (Chen et al., 2015). Downregulation of Nanog with siRNA has resulted in reduction of pluripotency markers such as Klf2 and Oct-4 in P19 stem cells, whereas expression of TOB1 has been upregulated by the Nanogsilencing (Choi et al., 2012). BTG1 appears to be required for maintaining stem cells quiescence and self-renewal (Farioli-Vecchioli et al., 2012). In addition, TIS21/BTG2 plays a pivotal role in maintaining the actual stem cells compartment in HSCs and hematopoiesis (Kim et al., 2008). BTG2 interacts with CAF1 deadenylase through its APRO domain, a defining feature of APRO family, to control cell proliferation (Stupfler et al., 2016). Long serial analysis of gene expression has suggested that TOB1 is differentially expressed during prenatal skeletal muscle development (Yuan et al., 2011). The expression of TOB1 increases with cellular senescence or aging, demonstrating that the 


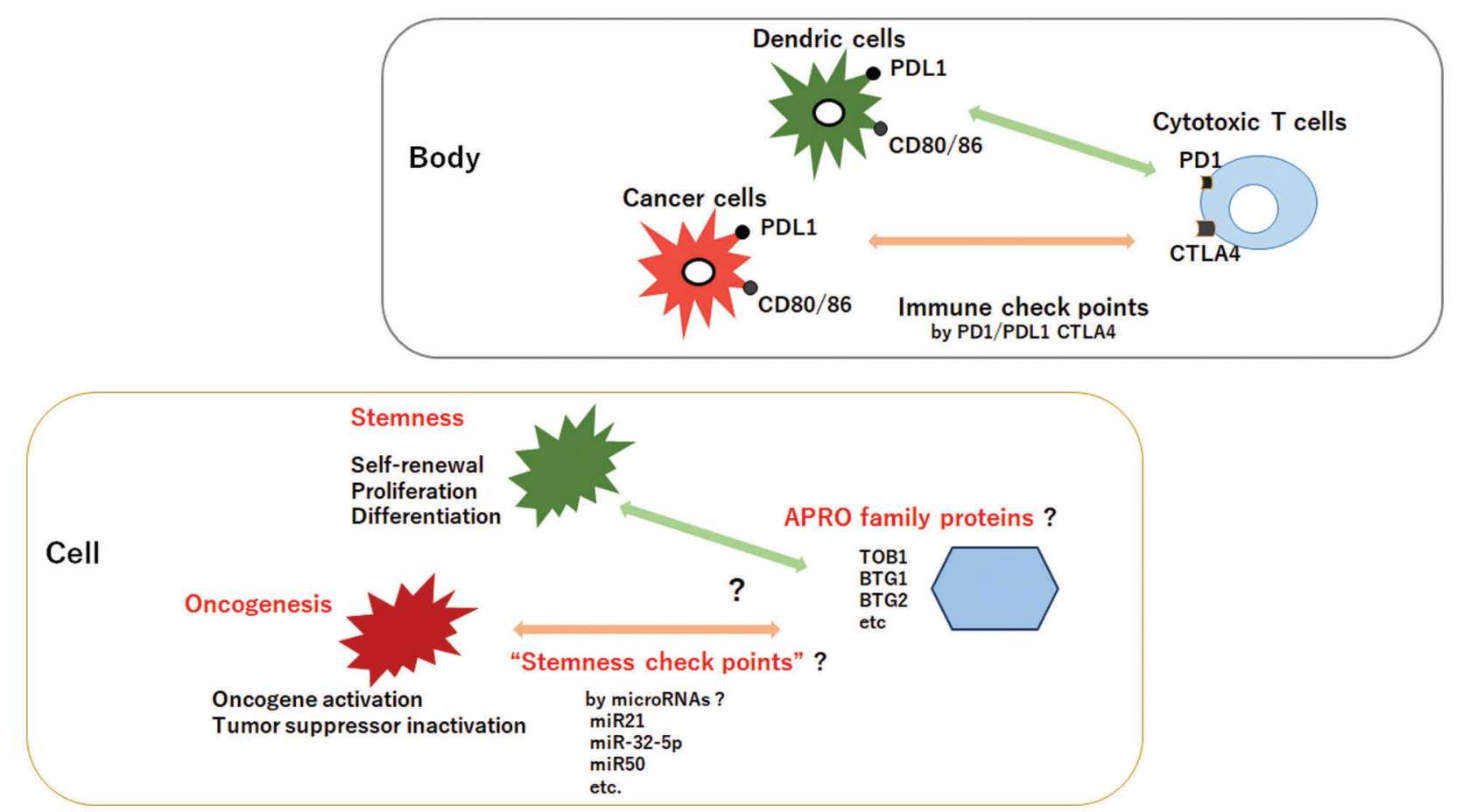

FIGURE 2. The relationship between cytotoxic T-cells and cancer-cells/dendritic-cells in a body resembles to the relationship between tumor suppressor APRO family proteins and oncogenesis/stemness-homeostasis in a cell. It could be hypothesized that the former is regulated with "immune checkpoints" molecules such as PD1/PDL1 and CTLA4, whereas the latter may be regulated by "stemness checkpoints" microRNAs for targeting APRO family proteins.

proliferation potential of myoblasts decreases along with the increase of TOB1 in postnatal muscle development (Yuan et al., 2011). In addition to affecting proliferation/differentiation during cellular development, all APRO family proteins might play an imperious role on keeping homeostasis in normal healthy cells but not in cancerous cells under a physiological condition in cellular stresses and/or carcinogenesis. It seems to be impossible that almost all cancer cells would differentiate without cancer-healing. For example, genomic profiling of B-cell leukemia and lymphoma has pointed BTG1 towards a role as a rising star of tumor suppressors (Moafi et al., 2017; Mao et al., 2015b), since BTG1 is frequently deleted or mutated in these malignancies (Moafi et al., 2017; Mao et al., 2015b). Furthermore, plasma exosomederived BTG-1 protein may be a potential biomarker for the prognosis in patients with non-small cell lung cancer (Wan et al., 2021). Tumor growth suppression by adenovirusmediated introduction of TOB1 in pancreatic cancer has been reported suggesting an application for chemotherapy-resistant cancer peritonitis (Yanagie et al., 2009). Problems of the engineered adenovirus vectors lie in the limited duration of target gene expression and the immunogenicity of the viruses. Artificial exosomes containing APRO proteins could suppress therapy-resistant cancer cell growth with less immunogenicity. The relationship between APRO molecules and carcinogenesis in a cell may be reminiscent of the relationship between cytotoxic $\mathrm{T}$ cells and cancer cells in a body called "immune check points". (Fig. 2) We would like to suggest here tentatively to use a term "stemness check points" for the former relationship. As mentioned above, we consider the "stemness check points" molecules seem to be certain miRNAs. In general, cancer cells might contain cancer stem cells. Hence, we suppose that the word "stemness check points" could be replaced with the word "onco-check points". These findings have suggested that APRO genes and/or proteins might work as potential implements for cancer therapy. However, it has to be confirmed accurately and would be a part of future intensive research.

Authors' Contributions: Conceptualization, Y.I. and S.M.; writing-original draft preparation, Y.I., N.N., A.T., K.T., Y.K. and S.M.; writing-review and editing, Y.I., N.N., A.T., K.T., Y.K. and S.M.; visualization, Y.I., N.N., A.T., K.T., Y.K. and S.M.; supervision, S.M. Each author has participated sufficiently in this work of drafting the article and/or revising the article for the important rational content. All authors have read and agreed to the published version of the manuscript.

Funding Statement: The authors received no specific funding for this study.

Conflicts of Interest: The authors declare that they have no conflicts of interest to report regarding the present study.

\section{References}

Barati M, Akhondi M, Mousavi NS, Haghparast N, Ghodsi A et al. (2021). Pluripotent stem cells: Cancer study, therapy, and vaccination. Stem Cell Reviews and Reports, 1-18. DOI 10.1007/s12015-021-10199-7 (Online ahead of print).

Bartel DP (2009). MicroRNAs: Target recognition and regulatory functions. Cell 136: 215-233. DOI 10.1016/j.cell.2009.01.002. 
Basalova N, Sagaradze G, Arbatskiy M, Evtushenko E, Kulebyakin K et al. (2020). Secretome of mesenchymal stromal cells prevents myofibroblasts differentiation by transferring fibrosis-associated microRNAs within extracellular vesicles. Cells 9: 1272. DOI 10.3390/cells9051272.

Chen J, Liu M, Luo X, Peng L, Zhao Z et al. (2020). Exosomal miRNA486-5p derived from rheumatoid arthritis fibroblast-like synoviocytes induces osteoblast differentiation through the Tob1/BMP/Smad pathway. Biomaterials Science 8: 34303442. DOI 10.1039/C9BM01761E.

Chen Y, Wang C, Wu J, Li L (2015). BTG/Tob family members Tob1 and Tob2 inhibit proliferation of mouse embryonic stem cells via Id3 mRNA degradation. Biochemical and Biophysical Research Communications 462: 208-214. DOI 10.1016/j. bbrc.2015.04.117.

Choi SC, Choi JH, Park CY, Ahn CM, Hong SJ et al. (2012). Nanog regulates molecules involved in stemness and cell cyclesignaling pathway for maintenance of pluripotency of P19 embryonal carcinoma stem cells. Journal of Cellular Physiology 227: 3678-3692. DOI 10.1002/jcp.24076.

Dexheimer PJ, Cochella L (2020). MicroRNAs: From mechanism to organism. Frontiers in Cell and Developmental Biology 8: 403. DOI 10.3389/fcell.2020.00409.

Farioli-Vecchioli S, Micheli L, Saraulli D, Ceccarelli M, Cannas S et al. (2012). Btg1 is required to maintain the pool of stem and progenitor cells of the dentate gyrus and subventricular zone. Frontiers in Neuroscience 6: 124. DOI 10.3389/fnins.2012.00124.

Gao Y, Zhang Y, Lu Y, Wang Y, Kou X et al. (2016). TOB1 deficiency enhances the effect of bone marrow-derived mesenchymal stem cells on tendon-bone healing in a rat rotator cuff repair model. Cellular Physiology and Biochemistry 38: 319329. DOI 10.1159/000438632.

Jiang S, Tian G, Li X, Yang Z, Wang F et al. (2021). Research progress on stem cell therapies for articular cartilage regeneration. Stem Cells International 2021: 8882505.

Kim BC, Ryu MS, Oh SP, Lim IK (2008). TIS21/(BTG2) negatively regulates estradiol-stimulated expansion of hematopoietic stem cells by derepressing Akt phosphorylation and inhibiting mTOR signal transduction. Stem Cells 26: 23392348. DOI 10.1634/stemcells.2008-0327.

Lanfredi GP, Thomé CH, Ferreira GA, Silvestrini VC, Masson AP et al. (2021). Analysis of ovarian cancer cell secretome during epithelial to mesenchymal transition reveals a protein signature associated with advanced stages of ovarian tumors. Biochimica et Biophysica Acta (BBA)-Proteins and Proteomics 1869: 140623. DOI 10.1016/j.bbapap.2021.140623.

Lang F, Liu Y, Chou FJ, Yang C (2021). Genotoxic therapy and resistance mechanism in gliomas. Pharmacology \& Therapeutics 228: 107922. DOI 10.1016/j.pharmthera.2021.107922.

Li YC, Xu FM, Zhang GQ, Li SB, Wen YY et al. (2018). Downregulation of microRNA-21 inhibits cell proliferation and invasion of high-invasion liver cancer stem cells. Europian Review for Medical and Pharmacol Sciences 22: 7832-7840.

Liang H, Tang Y, Zhang H, Zhang C (2019). MiR-32-5p regulates radiosensitization, migration and invasion of colorectal cancer cells by targeting TOB1 gene. Onco Targets and Therapy 12: 9651-9661. DOI 10.2147/OTT.

Liu T, Wu Y, Huang T, Zhang X, Cai Y (2017). miR-590 promotes the proliferation of HUMSCs and induces ECM synthesis by targeting Smad7. Oncology Letters 14: 3941-3946. DOI 10.3892/ol.2017.6663.
Mao B, Xiao H, Zhang Z, Wang D, Wang G (2015a). MicroRNA-21 regulates the expression of BTG2 in HepG2 liver cancer cells. Molecular Medicine Reports 12: 4917-4924. DOI 10.3892/ mmr.2015.4051.

Mao B, Zhang Z, Wang G (2015b). BTG2: A rising star of tumor suppressors (Review). International Journal of Oncology 46: 459-464. DOI 10.3892/ijo.2014.2765.

Matsuda S, Rouault J, Magaud J, Berthet C (2001). In search of a function for the TIS21/PC3/BTG1/TOB family. FEBS Letters 497: 67-72. DOI 10.1016/S0014-5793(01)02436-X.

Matsuda S, Nakagawa Y, Kitagishi Y, Nakanishi A, Murai T (2018). Reactive oxygen species, superoxide dimutases, and PTENp53-AKT-MDM2 signaling loop network in mesenchymal stem/stromal cells regulation. Cells 7: 36. DOI 10.3390/ cells7050036.

Micheli L, Ceccarelli M, Farioli-Vecchioli S, Tirone F (2015). Control of the normal and pathological development of neural stem and progenitor cells by the PC3/Tis21/Btg2 and Btg1 genes. Journal of Cellular Physiology 230: 2881-2890. DOI 10.1002/jcp.25038.

Moafi A, Zojaji A, Salehi R, Najafi Dorcheh S, Rahgozar S (2017). The correlation between Pax 5 deletion and patients survival in Iranian children with precursor B-cell acute lymphocytic leukemia. Cellular and Molecular Biology 63: 19-22. DOI 10.14715/cmb/2017.63.8.4.

Mohr AM, Mott JL (2015). Overview of microRNA biology. Seminars in Liver Disease 35: 3-11. DOI 10.1055/s-00000069.

Pasha A, Calvani M, Favre C (2021). Beta3-Adrenoreceptors as ROS balancer in hematopoietic stem cell transplantation. International Journal of Molecular Sciences 22: 2835. DOI 10.3390/ijms22062835.

Paul MK, Bisht B, Darmawan DO, Chiou R, Ha VL et al. (2014). Dynamic changes in intracellular ROS levels regulate airway basal stem cell homeostasis through Nrf2-dependent Notch signaling. Cell Stem Cell 15: 199-214. DOI 10.1016/j. stem.2014.05.009.

Priester C, MacDonald A, Dhar M, Bow A (2020). Examining the characteristics and applications of mesenchymal, induced pluripotent, and embryonic stem cells for tissue engineering approaches across the germ layers. Pharmaceuticals (Basel) 13: 344. DOI 10.3390/ph13110344.

Rajan TS, Giacoppo S, Diomede F, Ballerini P, Paolantonio M et al. (2016). The secretome of periodontal ligament stem cells from MS patients protects against EAE. Scientific Reports 6: 2329. DOI 10.1038/srep38743.

Stupfler B, Birck C, Séraphin B, Mauxion F (2016). BTG2 bridges PABPC1 RNA-binding domains and CAF1 deadenylase to control cell proliferation. Nature Communications 7: 67. DOI 10.1038/ncomms10811.

Treisman D, Li Y, Zhu Y (2021). Stem-like cell populations, p53-pathway activation and mechanisms of recurrence in sonic hedgehog medulloblastoma. NeuroMolecular Medicine. DOI 10.1007/s12017-021-08673-z. Online ahead of print.

Wan L, Chen X, Deng J, Zhang S, Tu F et al. (2021). Plasma exosome-derived B-cell translation gene 1: A predictive marker for the prognosis in patients with non-small cell lung cancer. Journal of Cancer 12: 1538-1547. DOI 10.7150/jca.52320.

Yanagie $H$, Tanabe T, Sumimoto $H$, Sugiyama $H$, Matsuda $S$ et al. (2009). Tumor growth suppression by adenovirus-mediated introduction of a cell growth suppressing gene tob in a 
pancreatic cancer model. Biomedicine \& Pharmacotherapy 63: 275-286. DOI 10.1016/j.biopha.2008.04.010.

Yang Y, Shen X, Sun K (2018). Prognostic value of transducer of ErbB2.1 (TOB1) expression in patients with gastric cancer: Tissue microarray analysis. International Journal of Clinical and Experimental Pathology 11: 4060-4066.

Yang C, Luo L, Bai X, Shen K, Liu K, Wang J, Hu D (2020). Highlyexpressed micoRNA-21 in adipose derived stem cell exosomes can enhance the migration and proliferation of the HaCaT cells by increasing the MMP-9 expression through the PI3K/AKT pathway. Archives of Biochemistry and Biophysics 681: 108259.
Yuan J, Cao JY, Tang ZL, Wang N, Li K (2011). Molecular characterization of Tob1 in muscle development in pigs. International Journal of Molecular Sciences 12: 4315-4326.

Yuniati L, Scheijen B, van der Meer LT, van Leeuwen FN (2019). Tumor suppressors BTG1 and BTG2: Beyond growth control. Journal of Cellular Biology 234: 5379-5389.

Zhang SQ, Yang Z, Cai XL, Zhao M, Sun MM, Li J, Feng GX, Feng JY, Ye LH, Niu JQ, Zhang XD (2017). miR-511 promotes the proliferation of human hepatoma cells by targeting the 3'UTR of B cell translocation gene 1 (BTG1) mRNA. Acta Pharmacologica Sinica 38: 1161-1170. 